\title{
Riqueza y abundancia de las aves urbanas de nueve áreas verdes de la ciudad de Sangolquí (Ecuador): Estudio preliminar
}

\section{Richness and abundance of the urban birds of the nine green ar- eas of Sangolquí (Ecuador): Preliminary study}

Iván Vinicio Jácome-Negrete ${ }^{1}$, Sofía Isabel Trujillo Regalado ${ }^{1}$, Diana Lizeth Rocha Cuascota ${ }^{1}$, Erika Andrea Hidalgo Cárdenas ${ }^{1 *}$, Stephanie Carolina Flores Vega

Autor para correspondencia: eahidalgoc@uce.edu.ec

Facultad de Ciencias Biológicas, Universidad Central del Ecuador, Iquique N 14-121 y Sodiro - Itchimbia, Quito - Ecuador

\begin{abstract}
Resumen
Este estudio se realizó con el objetivo de documentar la riqueza y la abundancia de las aves silvestres en nueve áreas verdes de Sangolquí. Se registró la avifauna mediante puntos de conteo a partir de las 06h30 hasta las 09h30, en días sin 1luvia, desde el 11 de noviembre del 2016 hasta el 18 de febrero del 2017, mediante detección visual y auditiva. Cada uno de los nueve sectores fue muestreado a través de siete puntos de conteo consecutivos. La avifauna registrada incluyó una riqueza de 29 especies con un total de 1.010 individuos registrados. La Tórtola Orejuda Zenaida auriculata fue la más abundante con 292 individuos, mientras que las especies menos abundantes, con un solo registro, fueron el Zorzal de Swainson Catharus ustulatus, Carpintero dorsicar mesi Colaptes rivolii, Gallinazo Negro Coragyps atratus, Pinchaflor Pechicanelo Diglossa sittoides y el Gavilán Alicastaño Parabuteo unicinctus. La Paloma Doméstica, el Gorrión, el Mirlo Grande y Tórtola Orejuda se consideran especies sinantrópicas para Sangolquí. Finalmente, se sugiere restaurar la cobertura arbustiva de las áreas verdes para favorecer la conservación de las aves locales.
\end{abstract}

Palabras clave: diversidad, áreas verdes urbanas, conservación, distribución, aves andinas.

\begin{abstract}
This study was done with the purpose of documenting the richness and abundance of wild birds in nine green areas of Sangolquí. Birdlife was recorded by counting points from 06.30 am to $09.30 \mathrm{am}$, on days without rain from November 11, 2016, until February 18, 2017, through visual and audio detection. Each of the nine sectors was sampled through seven consecutive counting points. The recorded birdlife included a wealth of 29 species with a total of 1.010 birds. The Eared Dove Zenaida auriculata was the most abundant with 292 birds, while the less abundant species, with only one record, were the Swainson's Thrush Catharus ustulatus, the Crimson-mantled Woodpecker Colaptes rivolii, the Black Vulture Coragyps atratus, the Rusty Flowerpiercer Diglossa sittoides and the Harris's Hawk Parabuteo unicinctus. The Rock Pigeon, the Rufous collared Sparrow, the Great Thrush, and the Eared Dove are considered synanthropic species for Sangolquí. Finally, it is suggested to restore the shrub cover of green areas to favor the conservation of local birds.
\end{abstract}

Keywords: diversity, urban areas, conservation, distribution, Andean birds. 


\section{Introducción}

Ecuador es considerado uno de los 17 países más biodiversos del mundo (Herrera \& Lasso, 2014) a pesar de su reducida extensión territorial, ocupa el primer lugar en número de especies por kilómetro cuadrado (García, Parra \& Mena, 2014). Esta diversidad tiene su origen en un conjunto de factores geográficos, geológicos y climáticos, entre los que destacan la presencia de la corriente fría de Humboldt y cálida del Niño, además de la existencia de la cordillera de los Andes (Mindo Cloudforest Fundation, 2010).

El grupo de vertebrados con mayor diversidad en el país corresponde a las aves que, según la versión actualizada del Listado de Aves del Ecuador, con los cambios taxonómicos del Comité de Clasificación de Sudamérica (SACC), comprende 1.684 especies (Freile, Brinkhuizen, Greenfield, Lysinger, Navarrete, Nilsson \& Boyla, 2018). De esta cifra, al menos 131 especies de aves de 34 familias y 15 órdenes han sido registradas para la zona urbana y periurbana de Quito, incluyendo los valles vecinos en los conteos navideños organizados con participación de la ciudadanía (Cisneros-Heredia, Amigo, Arias, Arteaga, Bedoya, Espinosa, Montenegro, Nazati \& Carrión, 2015).

Una parte de esta cifra se encuentra distribuida en la ciudad de Sangolquí, cabecera cantonal del cantón Rumiñahui, sitio ubicado en la provincia de Pichincha. Las aves del cantón han sido más estudiadas en áreas rurales, alejadas de la urbe. La última actualización publicada en cuanto al número de especies de aves registradas en este cantón fue hecha por Jácome \& Guarderas (2010), que indicaba la existencia de 48 especies de aves, agrupadas en 21 familias y 6 órdenes. En el caso específico de la ciudad de Sangolquí, no existe información publicada actualizada, sin embargo, se conoce que el número de especies, según los últimos censos publicados por Ebird en la web, es de 32 especies. Lara (2015) reporta la existencia de 61 especies de aves para todo el cantón Rumiñahui, como dato más actualizado.

En el país existen muchos lugares protegidos en áreas rurales, con el objetivo de promover la conservación de la avifauna, sin embargo, no es práctica cotidiana generar procesos de conservación en áreas urbanas, tales como los parques citadinos, al ser espacios que a pesar de la creciente antropización aún poseen especies nativas de la zona (Caula, Giner, \& De Nóbrega, 2010). Las zonas urbanas a pesar de la actividad antropogénica, albergan una alta riqueza de aves residentes y migratorias, usualmente más tolerantes a los disturbios generados por el ser huma- no que pueden ser objeto de atracción para personas avi-aficionadas (Jiménez Moreno \& Mendoza Cuamatzi, 2010). Las plazas y jardines de los ambientes urbanos favorecen la conservación de la diversidad de algunos grupos de aves, mientras tengan mayor cobertura vegetal natural (Maragliano, Lucas, Ibañez \& Montalti, 2009; Díaz \& Armesto, 2003).

Las áreas verdes de la ciudad de Sangolquí albergan varias especies de aves (Jácome \& Guarderas, 2010) y algunas de ellas pueden considerarse icónicas. Un ejemplo claro de ello es el monumento de "El Colibrí", que hace referencia al quinde Colibri coruscans, ave emblemática muy común para la ciudad y el cantón. En los entornos urbanos, los parques y su biodiversidad asociada, además, proporcionan importantes servicios ecosistémicos tales como la regulación del clima, la oferta de paisajes naturales y oportunidades de recreación (Balvanera, 2012). Reyes \& Gutiérrez (2010) y World Trade Organization (2010), mencionan que la calidad ambiental en una ciudad va a depender de la cantidad de árboles existentes, que mantengan la humedad del aire, de tal forma que las áreas verdes urbanas son vitales por los beneficios directos e indirectos que brindan a la comunidad, además de albergar especies de flora y fauna.

También, la observación de aves es una actividad económica sostenible en crecimiento para Ecuador. El aviturismo, como una actividad de observación de las aves con fines recreativos, no precisa manipulación o extracción de las aves de sus hábitats (Ben-Dalia, Collins-Kreiner \& Churchman, 2013), y su promoción al interior de la ciudad de Sangolquí podría favorecer simultáneamente la recreación familiar y la conservación biológica (Municipio de Rumiñahui, 2016). En Ecuador son varios los ejemplos en los que el aviturismo se ha convertido en una actividad económica importante que une la recreación y la conservación, en zonas del noroccidente de Pichincha, Loja y Zamora y ciertas áreas de la Costa y Amazonía (Abril \& Adela, 2011). Últimamente está actividad inclusive se ha promocionado notablemente a nivel urbano, a través de los conteos navideños que se hacen en varias ciudades andinas cada año.

En este contexto, el presente trabajo se realizó con el objetivo de cuantificar la riqueza y abundancia de las aves de nueve áreas verdes de Sangolquí, para ampliar la escasa información sobre la diversidad de las aves existentes en esta área urbana. Se espera también sugerir acciones que favorezcan su conservación y la incursión futura en actividades más sostenibles como el aviturismo urbano. 


\section{Materiales y Métodos}

\section{1 Área de estudio}

El muestreo se realizó al interior de nueve áreas verdes, ubicadas desde el redondel de "El Choclo" hasta el redondel de "San Luis", en la ciudad de Sangolquí, cantón Rumiñahui (Pichincha, Ecuador), correspondiente al Piso Zoogeográfico Templado (Albuja, Alméndariz, Barriga, Montalvo, Cáceres \& Román, 2012). Por su composición botánica, según Sierra (1999), la zona pertenece a la formación Matorral húmedo montano del sector norte y centro de los valles interandinos, aunque la mayor parte de la vegetación natural ha sido reemplazada por especies exóticas ornamentales.

Para el estudio fueron seleccionados nueve sitios dentro de las áreas verdes que bordean al río
Santa Clara: 1) Parque El Ejido, 2) Parque lineal Santa Clara (PLSC) sector "El Choclo", 3) PLSC sector Hospital, 4) PLSC sector Pista de Patinaje, 5) PLSC sector River Mall, 6) PLSC sector Pista Atlética, 7) PLSC sector Colegio Henry Dunant, 8) Parque La Alegría y 9) PLSC sector San Luis (Figura 1). La vegetación de los nueve sectores contiene los estratos herbáceo, arbustivo y dosel, constituidos por una mixtura de especies ornamentales introducidas en su mayor parte, y nativas de los Andes. El estrato herbáceo está dominado por Pennisetum clandestinum y otras poáceas nativas. El estrato arbustivo incluyó principalmente especies típicas de matorral húmedo de las familias Melastomataceae, Scrophulariaceae, Solanaceae y Verbenaceae, mientras que el dosel de los parques estuvo mayormente representado por eucaliptos Eucalyptus sp., pino Pinus sp., ciprés Cupressus sp., sauce Salix sp. y acacias Acacia sp., entre otros.

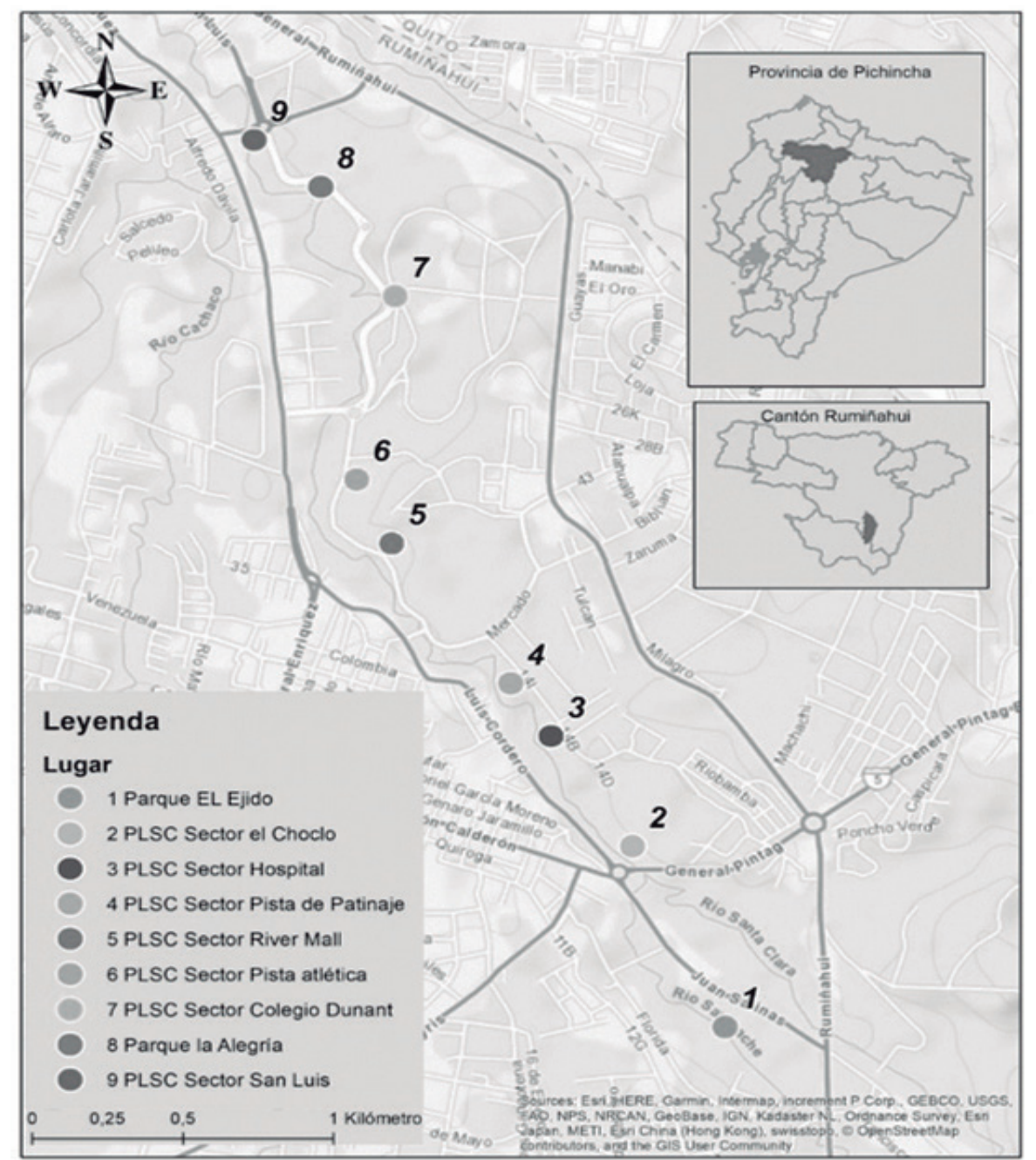

Figura 1. Área de estudio (Fuente: Esri, HERE, Garmin, Intermap, increment P Corp., GEBCO, USGS, FAO, NPS, NRCAN, GeoBase, IGN, Kadaster NL, Ordnance Survey, Esri Japan, METI, Esri China (Hong Kong), swisstopo, (C) OpenStreetMap contributors, and the GIS User Community) 


\subsection{Inventario de las aves}

Para documentar la riqueza y abundancia de las aves asociadas a los sectores de muestreo se utilizó la metodología de puntos de conteo (Ralph, Geupel, Pyle, Martin, De Santé \& Milá (1996). Esta metodología ha sido ampliamente usada en ecosistemas naturales y antropizados del neotrópico para estimar la riqueza, abundancia, composición de especies, índices de importancia y elaborar listados ecológicos (Cerezo, Robbins \& Dowell, 2008). En las ciudades, este método ha permitido cuantificar la diversidad de aves y correlacionarla con la mayor o menor cobertura, considerando a las áreas verdes como fragmentos de vegetación natural (Malagamba-Rubio, MacGregor-Fors \& Pineda-López, 2013).

En cada sector se estableció un punto de conteo circular con una superficie de muestreo de $2.826 \mathrm{~m}^{2}($ radio $=25 \mathrm{~m})$. Dentro de la superficie de muestreo se contabilizaron todas las aves observadas y detectadas auditivamente durante un lapso de diez minutos. Los puntos de conteo se iniciaron a partir de las 06h30 hasta las 09h30, en días sin lluvia, desde el 11 de noviembre del 2016 hasta el 18 de febrero del 2017. Los registros realizados incluyeron los siguientes datos: la identidad taxonómica del ave a nivel de especie, la frecuencia de individuos avistados, el comportamiento observado, el sustrato y el estrato de percha. Para la observación de las aves se usaron un par de binoculares Carton $8 \times 30$ Field $7.5^{\circ}$ y un monocular BAK4/FMC $12 \times 50$. Las aves fueron identificadas por contraste visual con las guías de aves de McMullan \& Navarrete (2013), Ridgely \& Greenfield (2006) y Jácome \& Guarderas (2010).

En total se establecieron 63 puntos de conteo durante el inventario realizado con un total de 10,5 horas de registro. Cada uno de los nueve sectores fue muestreado a través de siete puntos de conteo (70 minutos de registro).

\subsection{Análisis estadístico}

A partir de las frecuencias de registro de cada especie se organizó una matriz de datos usando como muestras a cada punto de conteo realizado. A partir de esta matriz fue posible proyectar la curva de acumulación de especies cuyo eje de las $x$ corresponde al número de especies y el eje de las $y$ expresa el esfuerzo de muestreo; además se ge- neró una curva de estimadores no paramétricos de la riqueza, como recomienda Villarreal, Álvarez, Córdova, Escobar, Fagua, Gast, Mendoza, Ospina \& Umaña (2006). El estimador no paramétrico de especies utilizado fue Chao 1, que fue calculado con el programa estadístico EstimateS versión 7 (Colwell, 2009), con las opciones preestablecidas del mismo.

Para conocer el porcentaje de cada gremio trófico encontrado en el estudio se realizó una regla de tres simple, tomando en cuenta el número total de individuos y el número de individuos de cada uno de los siete gremios tróficos.

El cálculo de la completitud es un análisis sencillo, útil para verificar si ha sido suficiente el esfuerzo de muestreo y se lo realizó mediante regla de tres simple. Según Martella, Trumper, Bellis, Renison, Giordano, Bazzano \& Gleiser (2012), para determinar que un muestro es eficiente se debe alcanzar al menos un $85 \%$ de completitud estimada.

Se realizó además una curva de rango-abundancia para determinar la estructura de la comunidad de aves estudiada. Las especies fueron ordenadas de mayor a menor abundancia y, con base en esta disposición, se obtuvo un perfil de abundancias por rango. En estas curvas, las abundancias se representan en escala logarítmica (en este caso base 10) y presentan la enorme ventaja de facilitar el resumen de gran cantidad de información de interés biológico como indica Whittaker (1972).

\section{Resultados}

Durante el estudio se registraron un total de 1.010 individuos que corresponden a 29 especies de aves en los 9 sitios del sector de Sangolquí, muestreados. La tórtola Zenaida auriculata fue la especie más abundante con un total de 292 individuos, seguida por la golondrina azuliblanca Pygochelidon cyanoleuca con 221 individuos, el mirlo Turdus fuscater con 164 individuos y el quinde herrero Colibri coruscans con 108 individuos (Tabla 1, Anexo 1). Mientras que las especies menos abundantes, con un solo registro fueron el zorzal de Swainson Catharus ustulatus, carpintero dorsicarmesí Colaptes rivolii, gallinazo negro Coragyps atratus, pinchaflor pechicanelo Diglossa sittoides y gavilán alicastaño Parabuteo unicinctus (Anexo 1). 
Tabla 1. Familias y especies encontradas con su respectivo gremio trófico y respectivas abundancias

\begin{tabular}{|c|c|c|c|}
\hline Familia/Nombre científico & Nombre común & Gremio trófico & Abundancia \\
\hline \multicolumn{4}{|l|}{ Ardeidae } \\
\hline Bubulcus ibis & Garza bueyera & Insectívoro & 3 \\
\hline \multicolumn{4}{|l|}{ Cathartidae } \\
\hline Coragyps atratus & Gallinazo negro & Carroñero & 1 \\
\hline \multicolumn{4}{|l|}{ Accipitridae } \\
\hline \multirow[t]{2}{*}{ Parabuteo unicinctus } & Gavilán alicastaño & Carnívoro & 1 \\
\hline & Falconidae & & \\
\hline Falco sparverius & Quilico & Carnívoro & 10 \\
\hline \multicolumn{4}{|l|}{ Columbidae } \\
\hline Zenaida auriculata & Tórtola & Granívoro & 292 \\
\hline Columba livia & Paloma & Granívoro & 26 \\
\hline Patagioenas fasciata & Torcaza & Granívoro & 7 \\
\hline \multicolumn{4}{|l|}{ Trochilidae } \\
\hline Colibri coruscans & Quinde herrero & Nectarívoro & 108 \\
\hline Lesbia victoriae & Colacintillo colinegro & Nectarívoro & 2 \\
\hline Chlorostilbon melanorhynchus & Esmeralda occidental & Nectarívoro & 2 \\
\hline \multicolumn{4}{|l|}{ Apodidae } \\
\hline Streptoprocne zonaris & Vencejo cóndor & Insectívoro & 3 \\
\hline \multicolumn{4}{|l|}{ Picidae } \\
\hline Colaptes rivolii & Carpintero Dorsicarmesí & Insectívoro & 1 \\
\hline \multicolumn{4}{|l|}{ Furnariidae } \\
\hline Synallaxis azarae & Colaespina de Azara & Insectívoro & 7 \\
\hline \multicolumn{4}{|l|}{ Tyraniidae } \\
\hline Sayornis nigricans & Febe guardarríos & Insectívoro & 16 \\
\hline Pyrocephalus rubinus & Pájaro brujo & Insectívoro & 5 \\
\hline Anairetes parulus & Cachudito torito & Insectívoro & 4 \\
\hline Elaenia albiceps & Elenia crestiblanca & Insectívoro & 2 \\
\hline Camptostoma obsoletum & Mosquerito silbador & Insectívoro & 2 \\
\hline \multicolumn{4}{|l|}{ Turdidae } \\
\hline Turdus fuscater & Mirlo & Omnívoro & 164 \\
\hline Catharus ustulatus & Zorzal de Swainson & Frugívoro & 1 \\
\hline
\end{tabular}


Mimidae

Mimus gilvus

Hirundinidae

Pygochelidon cyanoleuca

Orochelidon murina

Emberizidae

Zonotrichia capensis

Thraupidae

Thraupis episcopus

Pipraeidea bonariensis

Diglossa sittoides

Cardinalidae

Pheuticus chrysogaster

Fringillidae

Euphonia cyanocephala
Sinsonte tropical

Golondrina Azuliblanca

Golondrina ventricafé

Gorrión

Tangara azuleja

Tangara azuliamarilla

Pinchaflor Pechicanelo

Huiracchuro

Eufonia lomidorada
Omnívoro

Insectívoro

Insectívoro

8

Granívoro

Frugívoro

4

Frugívoro

2

Nectarívoro

1

Granívoro

35

Frugívoro 33

1.010
El gremio trófico mejor representado fue el gremio granívoro con el 39.9\%, y la especie que más aportó a este porcentaje fue Zenaida auriculata. A continuación, sigue el gremio insectívoro, luego el gremio omnívoro con sus especies más re- presentativas tales como Pygochelidon cyanoleuca y Turdus fuscater, respectivamente (Tabla 1). Los grupos menos abundantes fueron los carroñeros $\mathrm{y}$ carnívoros con el $0.1 \%$ y $1.1 \%$ como se muestra en la tabla 2.

Tabla 2. Porcentaje de los gremios tróficos

\begin{tabular}{ccc}
\hline Gremios Tróficos & Número de individuos & \% Total \\
\hline Carroñeros & 1 & 0.1 \\
Carnívoros & 11 & 1.1 \\
Frugívoros & 40 & 4.0 \\
Nectarívoros & 113 & 11.2 \\
Omnívoros & 170 & 16.8 \\
Insectívoros & 272 & 26.9 \\
Granívoros & 403 & 39.9 \\
\hline Total & $\mathbf{1 0 1 0}$ & $\mathbf{1 0 0 . 0}$ \\
\hline
\end{tabular}

La figura 2 muestra la curva de acumulación de especies asintóticas con un total de 29 especies mientras que con el estimador estadístico Chao 1 predice una cifra de 31 especies de aves potencial- mente existentes en los sitios de muestreo. Se estimó la completitud alcanzada en un $93.54 \%$, por lo que podemos afirmar que el muestreo de las aves fue adecuado. 


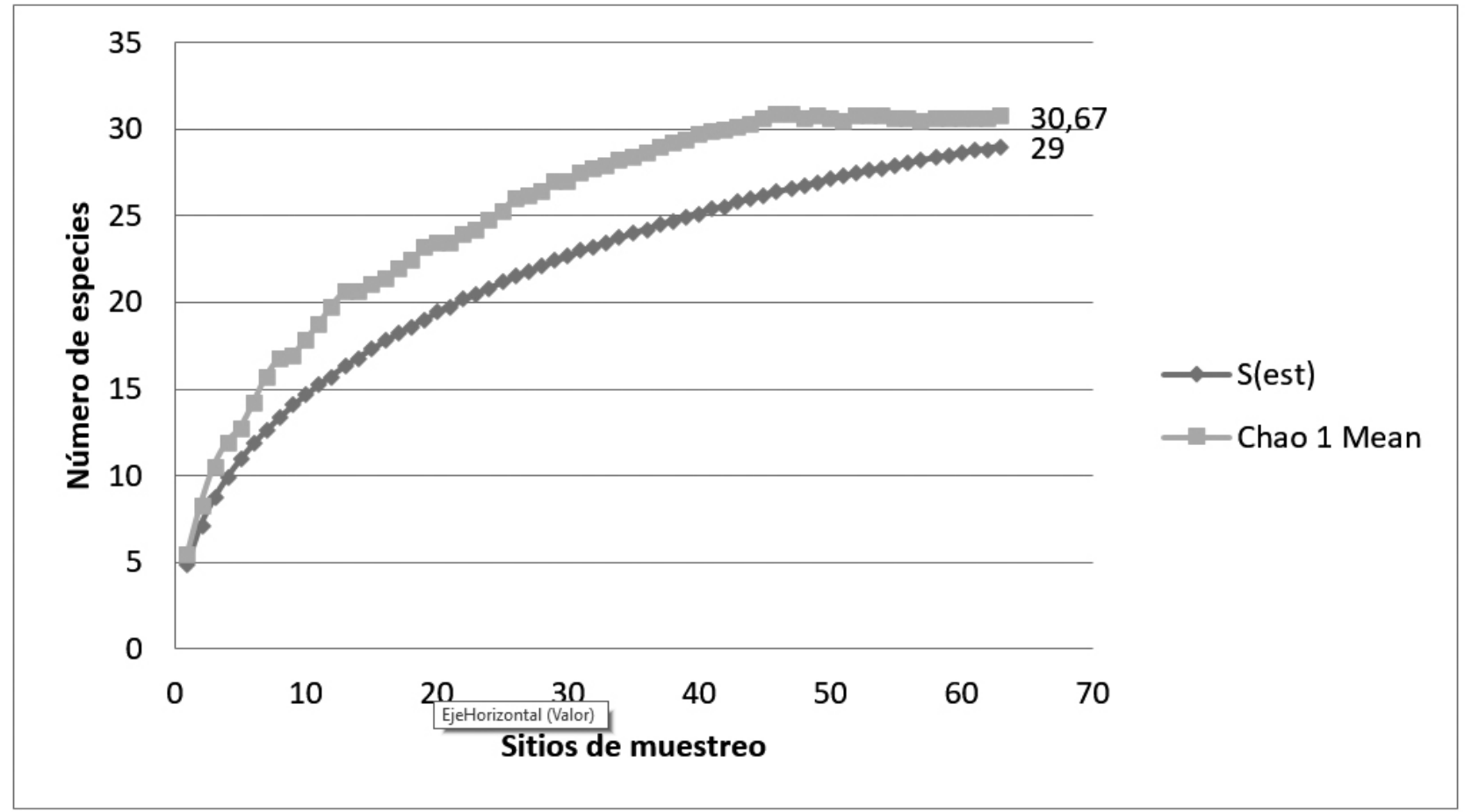

Figura 2. Riqueza potencial de aves en los sitios de muestreo.

Como se muestra en la figura 3, las familias más abundantes durante el estudio fueron Columbidae, Hirundinidae, Turdidae y Trochilidae, mientras que las menos abundantes fueron Picidae y Accipitridae con un solo individuo para cada familia.

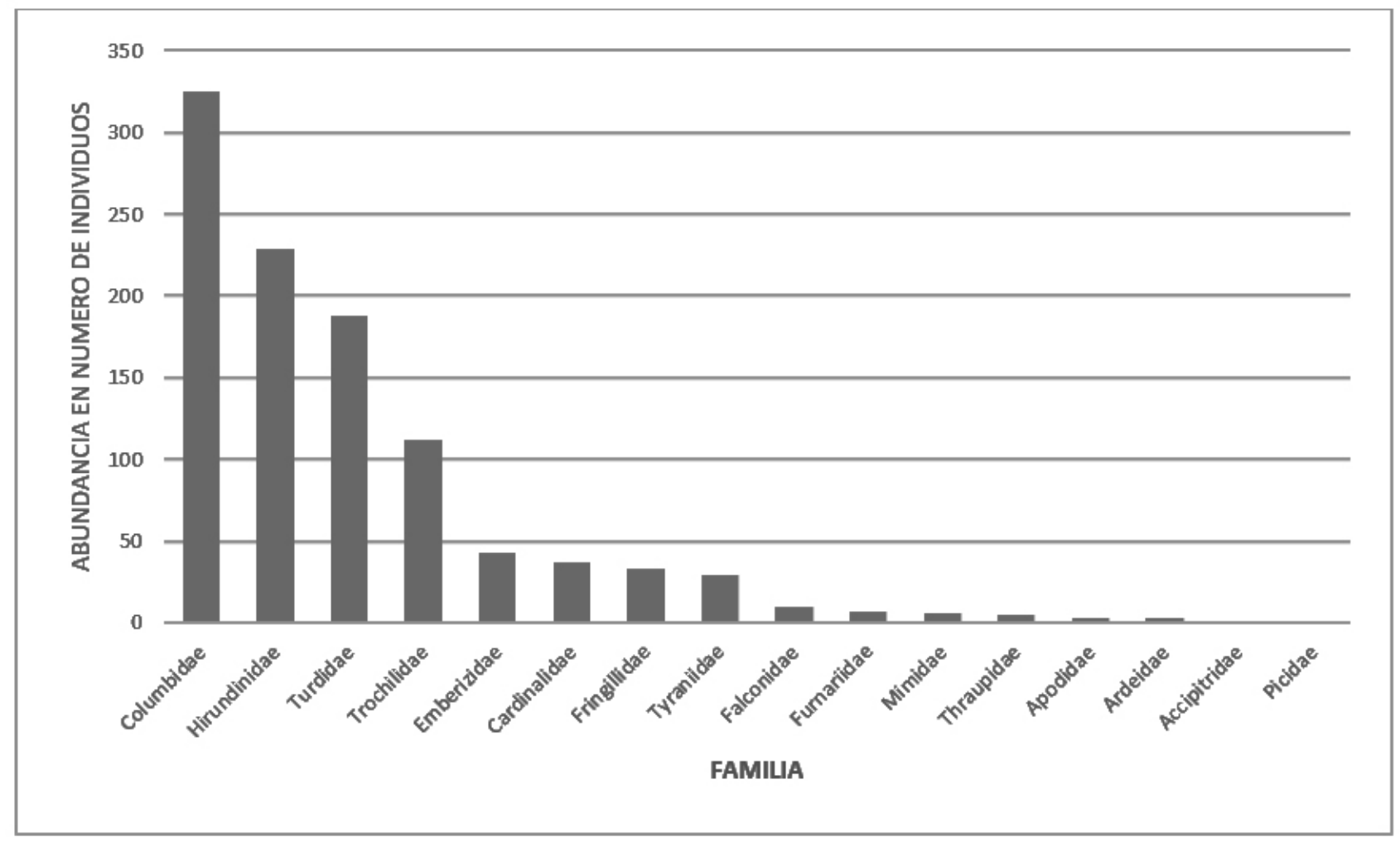

Figura 3. Abundancia de individuos por familias zoológicas. 
En cuanto a la riqueza de especies de la figura 4, la familia predominante fue Tyraniidae con 5 especies, seguida de Columbidae, Thraupidae y Tro- chilidae con 3 especies para cada familia mientras que las familias restantes únicamente registraron 1 o 2 especies.

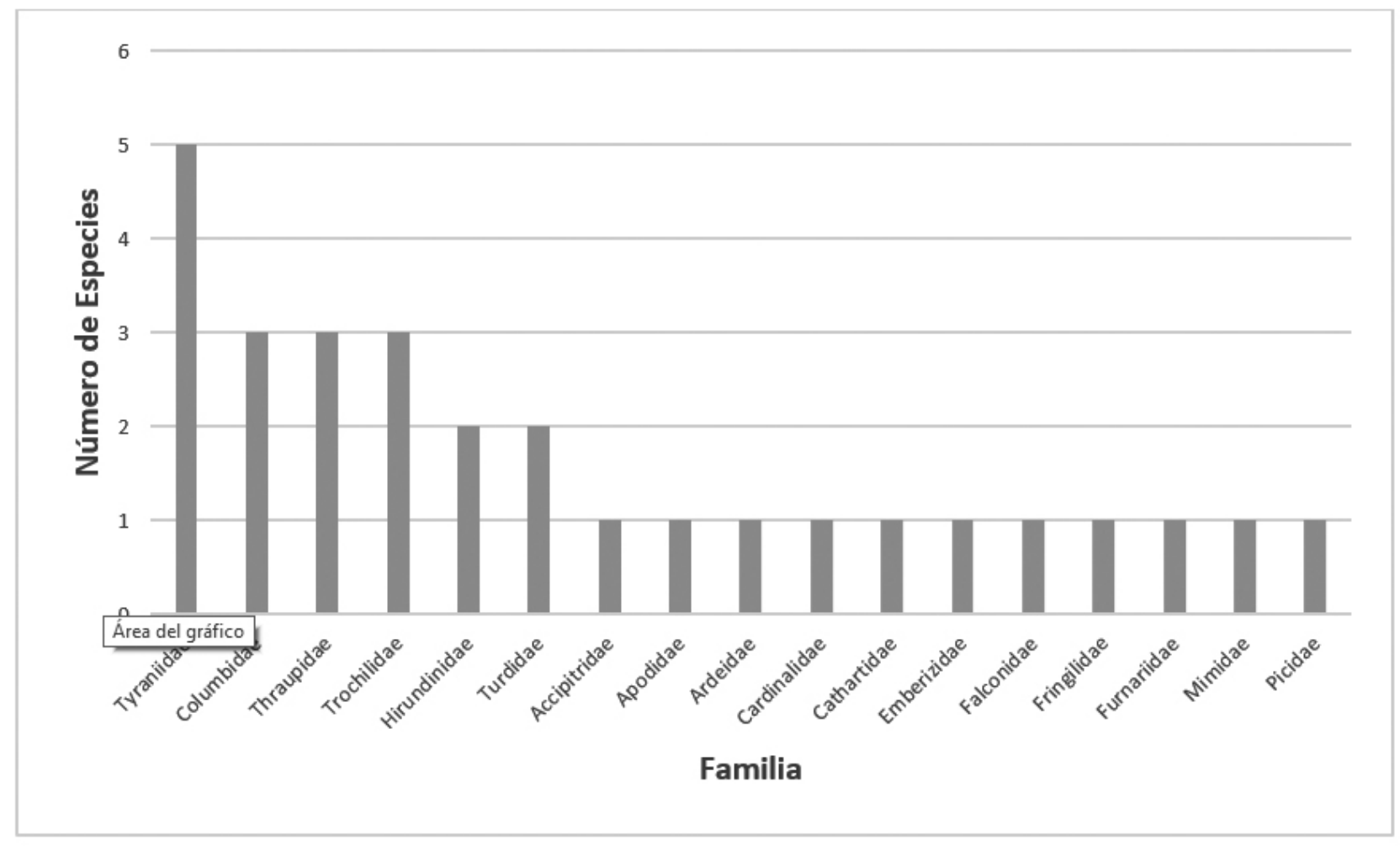

Figura 4. Riqueza de especies por familia.

Las especies más abundantes fueron Zenaida auriculata, Pygochelidon cyanoleuca, Turdus fuscater y Colibri coruscans, mismas que son relativamente fáciles de observar en los sectores de muestreo, a diferencia de especies muy raras como Catharus ustulatus, Colaptes rivolii, Coragyps atratus y Parabuteo unicinctus como se muestra en la figura 5.

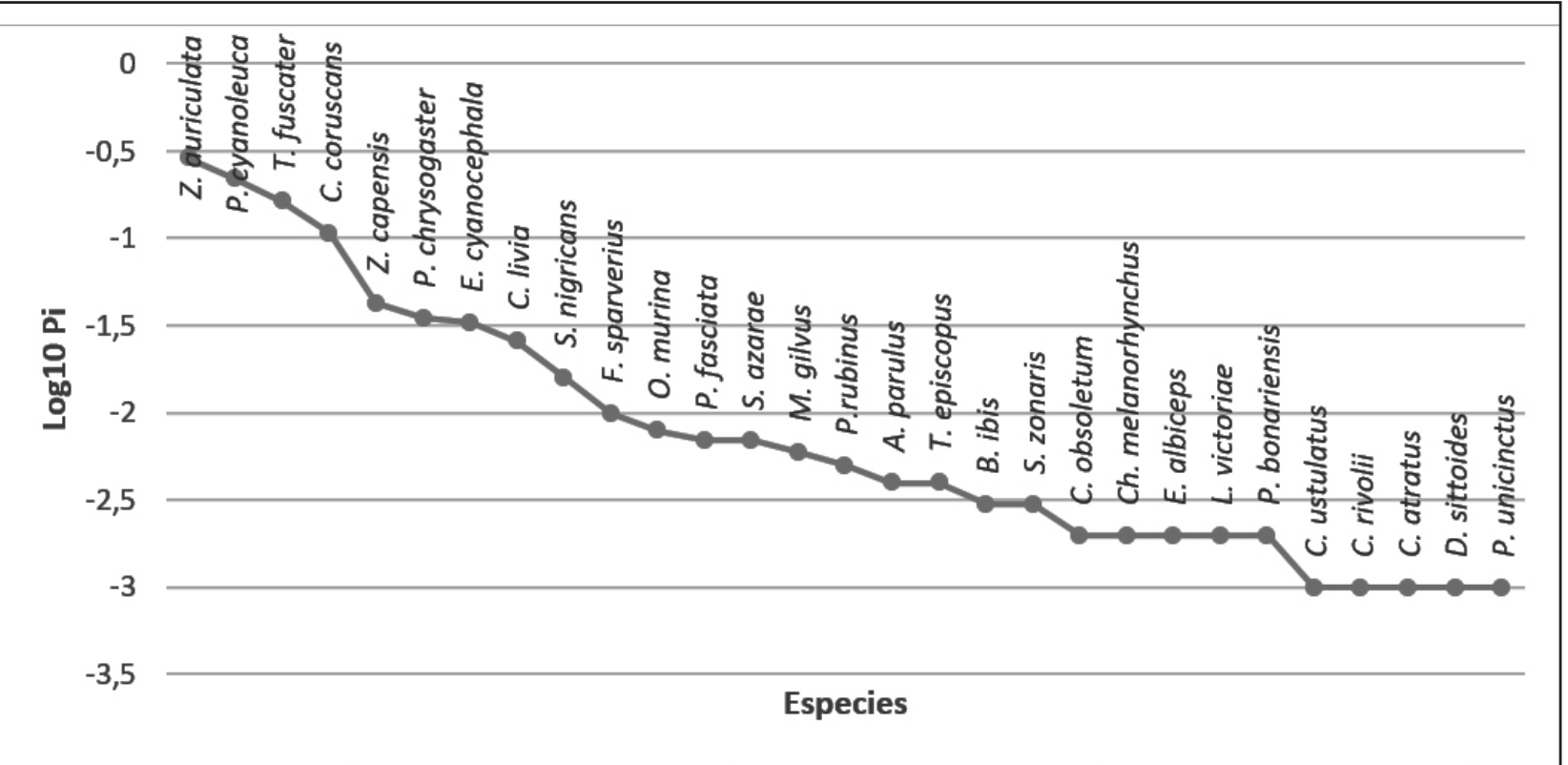

Figura 5. Curva de rango-abundancia o de Whittaker. 


\section{Discusión}

Los parques de Sangolquí representan áreas importantes para la supervivencia de las aves locales, abarcando entre 8 y 13 especies en cada parque, con un total de 29 especies registradas, cifra relativamente alta si se contrasta con el estudio realizado por Montenegro-Pazmiño (2015) sobre la diversidad de aves en áreas verdes de la ciudad de Quito, en donde se encontraron 59 especies, tomando en cuenta que los parques muestreados en Quito presentan extensiones mucho más grandes como es el caso de los parques La Carolina, Metropolitano y Las Cuadras.

Existen factores que influyen directamente sobre variaciones de diversidad en la avifauna, como la altura a nivel del mar (Blake \& Loiselle, 2000), factor ligado a la complejidad estructural vegetal del sitio. Garitano-Zavala \& Gismondi (2003) mencionan que la cobertura vegetal evidencia con mayor claridad esta variación faunística, ya que ambos factores señalados determinan una mayor diversidad de recursos alimenticios y hábitats usados por las aves, debido a que la distribución de las especies obedece a cambios en la vegetación y a movimientos temporales de las aves modificados según la disponibilidad del alimento (Ramírez-Albores, 2006). En un estudio realizado por Blake \& Loiselle (2001) se concluyó que a medida que las tasas de producción de follaje, flores y frutos en ecosistemas son más altas, la riqueza y abundancia avifaunística también incrementarán. El análisis general de la vegetación a lo largo del estudio ayuda mucho a explicar la presencia o ausencia, aumento o declive de ciertas especies. El área de estudio posee tres estratos vegetales (herbáceo, arbustivo y dosel), cada uno con sus respectivas características, y factores que favorecen la presencia de ciertas especies dentro del área.

Las especies sinantrópicas encontradas en este trabajo fueron Zenaida auriculata, Pygochelidon cyanoleuca, Turdus fuscater, Colibri coruscans y Zonotrichia capensis, que son generalistas y no requieren de hábitats muy específicos. Estas especies concuerdan con las encontradas por Garitano-Zavala \& Gismondi (2003) en su estudio desarrollado en las áreas verdes urbanas de las ciudades de La Paz y El Alto (Bolivia) y con el de Montenegro-Pazmiño (2015), donde se reporta que las especies poco comunes se encuentran en parques que contienen pequeños parches de matorral o bosque que pueden proporcionar otro tipo de recursos para aquellas aves menos frecuentes. Dentro de las especies raras encontradas en los sectores estudiados se registró a Catharus ustulatus, Colaptes rivolii, Coragyps atratus y Parabuteo unicinctus, especies que poseen un extenso rango migratorio o son de amplia dispersión (Ridgely \& Greenfield, 2006) y que usualmente son raras de registrar. La falta de datos específicos de la preferencia de hábitat de cada individuo registrado y la variación de la riqueza en cada uno de los sitios de estudio podría ser explicada en términos tróficos.

Los análisis realizados muestran que se alcanzó un $93.54 \%$ de todas las especies esperadas en el área, lo que hizo de este trabajo un estudio representativo, dado que el esfuerzo de muestreo ha sido suficiente para registrar el número de especies potencialmente existentes para los parques de Sangolquí; mientras que la curva de rango-abundancia o de Whittaker muestra la existencia de numerosas especies codominantes y pocas especies raras, en concordancia con lo mencionado en el estudio de Trávez \& Yánez (2017), acerca de la diversidad y abundancia de avifauna urbana en el campus de la UIDE y el Parque Metropolitano Guangüiltagua, como áreas verdes igualmente antropizadas.

Los gremios tróficos encontrados responden a los diferentes recursos disponibles en cada uno de los parques. Al parecer, las especies sinantrópicas como Zenaida auriculata, Zonotrichia capensis, Pheuticus chrysogaster, Columba livia (granívoras), Pygochelidon cyanoleuca (insectívora), Turdus fuscater (omnívora), Colibri coruscans (nectarívoro) y Falco sparverius (carnívoro) son las que más aportan en el porcentaje total encontrado para su respectivo gremio trófico (Montenegro-Pazmiño, 2015). El bajo porcentaje de carnívoros y carroñeros podría estar relacionado a ciertos factores como la antropización del área de los parques, requerimientos de presas más grandes y la biología propia de estas especies (Berget, 2006).

Varias especies como Euphonia cyanocephala (frugívora), Chlorostilbon melanorhynchus y Diglossa sittoides (nectarívoros) están más asociados a un tipo de vegetación arbustiva y aprovechan estos pequeños parches de matorrales para su forrajeo (Arteaga, 2017). Finalmente, la diversidad de gremios tróficos aporta al equilibrio ecosistémico y dicha diversidad puede variar dependiendo de la fenología de las especies vegetales durante el año. 


\section{Conclusiones}

Estudios como el realizado son importantes ya que permiten entender cómo los parques urbanos son espacios importantes que contribuyen a la riqueza y abundancia de las aves en las ciudades. Adicionalmente a ello, es fundamental tomar en cuenta variables tales como el tipo de vegetación y la edad de la misma, que proporcionan sustento a la avifauna local proveyendo sustratos de nidificación y recursos alimenticios (René, Marti, Ibáñez \& Montalti, 2009). Otro factor de análisis a considerar es el reemplazo o eliminación de la vegetación natural que incrementa en las ciudades las poblaciones de aves oportunistas, sean éstas introducidas o nativas sinantrópicas. En el caso de este estudio, las especies sinantrópicas fueron Columba livia, Zonotrichia capensis, Turdus fuscater y Zenaida auriculata, que han sido registradas prácticamente en todas las áreas verdes prospectadas y que además son las especies únicas o dominantes en la mayoría de las zonas de registro, al igual que lo encontrado en otras ciudades andinas (Garitano-Zavala \& Gismondi, 2003).
En este contexto, se considera fundamental que en el diseño, implementación o readecuación de los parques urbanos de Sangolquí, se considere la conservación o restauración del paisaje natural, manteniendo intacta la vegetación boscosa y arbustiva. Este tipo de desarrollo ayudaría a conservar e incrementar la valiosa biodiversidad avifaunística existente, la que constituye un valor recreativo agregado para los parques urbanos (Caula, Giner \& de Nóbrega (2010). La promoción de la conservación a largo plazo de las aves urbanas encontradas permitiría promover el conocimiento de la avifauna autóctona de parte de los habitantes del cantón Rumiñahui y generar inclusive opciones novedosas de aviturismo urbano.

\section{Agradecimientos}

Agradecemos a Carlos Andrés Valle por facilitarnos el mapa del área de estudio, a Nelson Monteros por colaborar con la fotografía de Catharus ustulatus incluida en el catálogo fotográfico, y a Iván Jácome Guarderas por su participación durante la fase de campo del presente estudio.

\section{Anexos}

Anexo 1. Aves comunes y raras de las áreas verdes de Sangolquí, Ecuador. Fotos: Iván Jácome Negrete (IJ); Diana Rocha (DR); Nelson Monteros(NM).

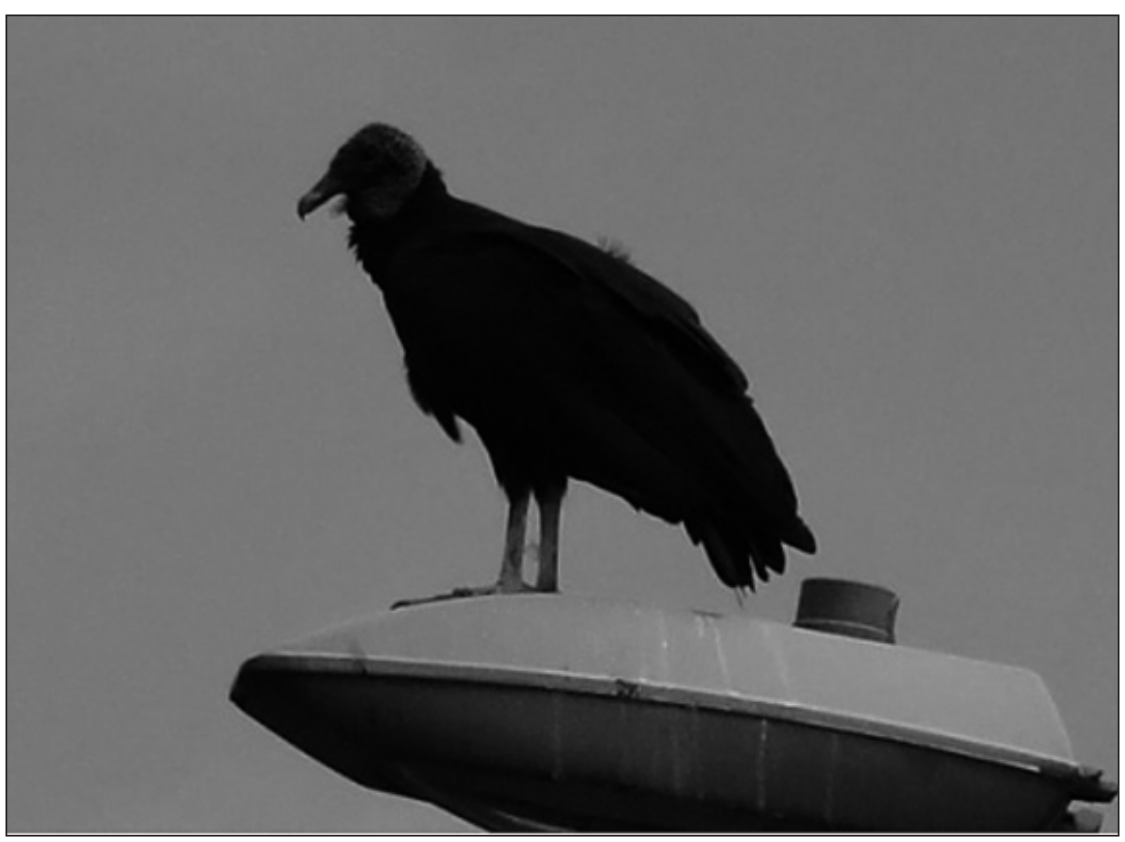

1. Coragyps atratus (IJ)

Gallinazo negro CATHARTIDAE

Raro 


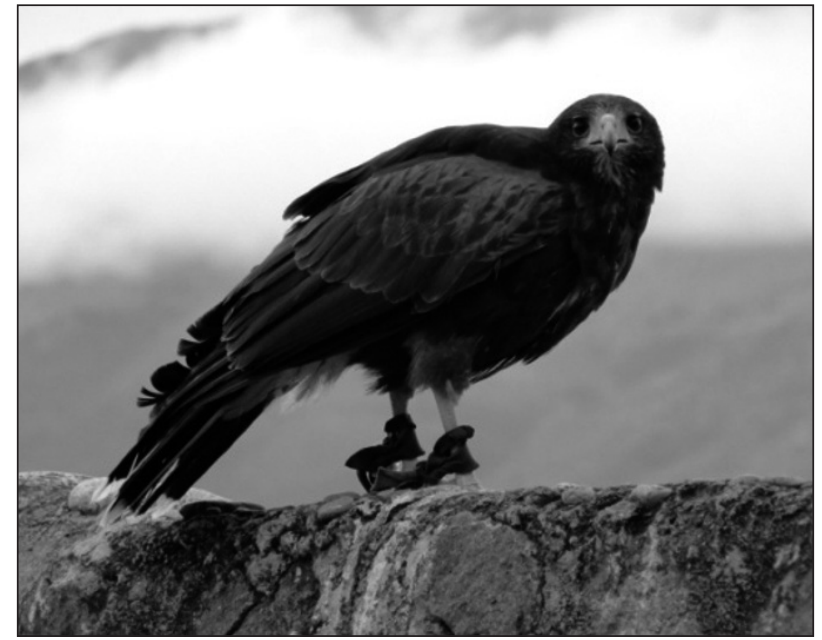

2. Parabuteo unicinctus (IJ)

Gavilán alicastaño ACCIPITRIDAE

Raro

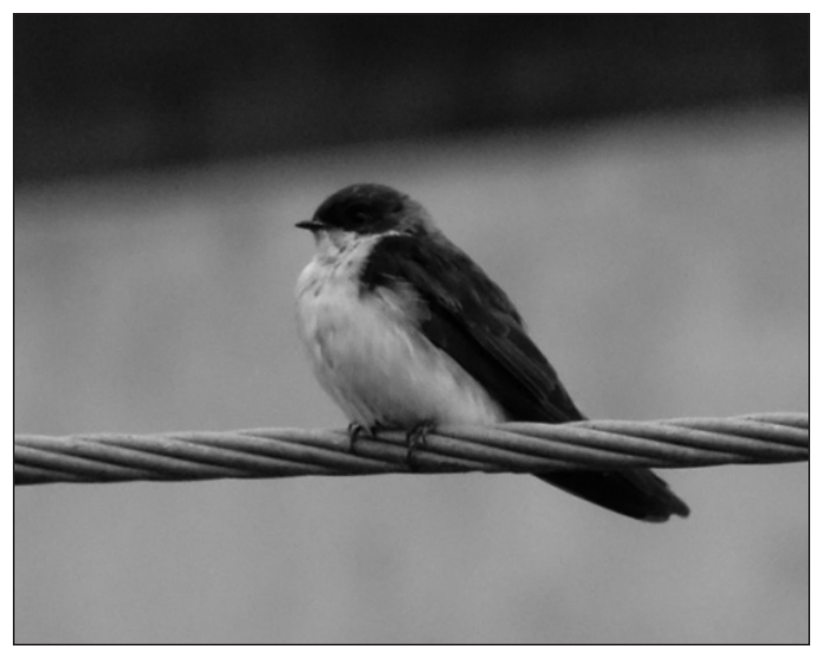

\section{Pygochelidon cyanoleuca (IJ)}

Golondrina azuliblanca HIRUNDINIDAE

Común

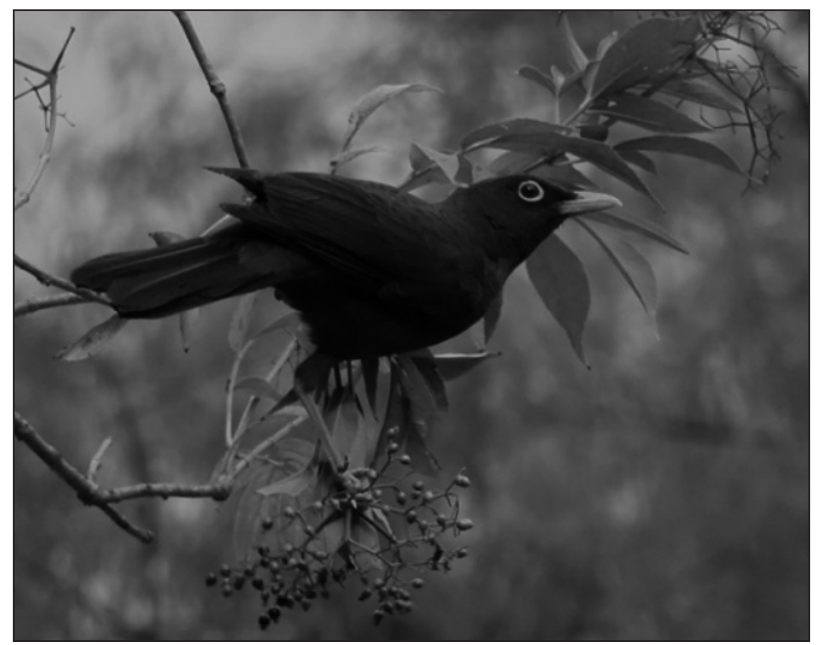

\section{Turdus fuscater (IJ)}

Mirlo TURDIDAE

Común

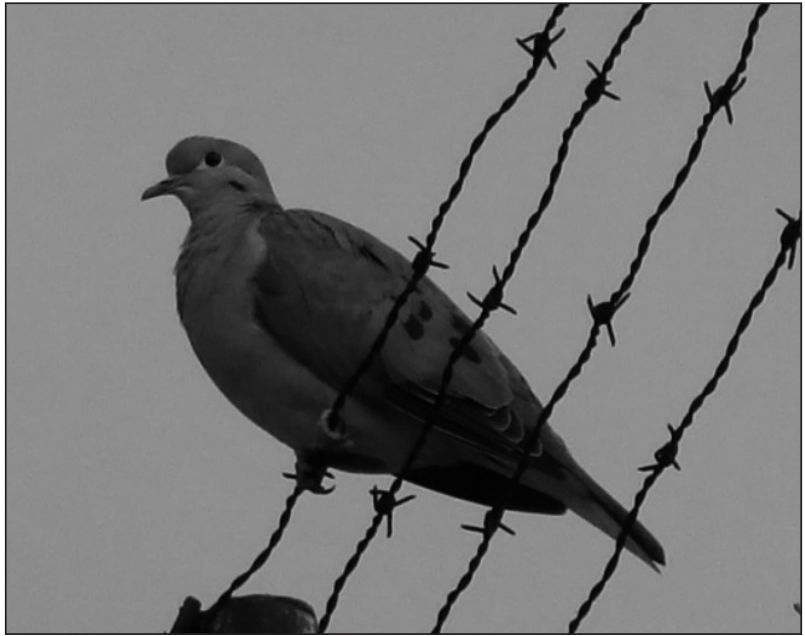

3. Zenaida auriculata (IJ)

Tórtola COLUMBIDAE

Común

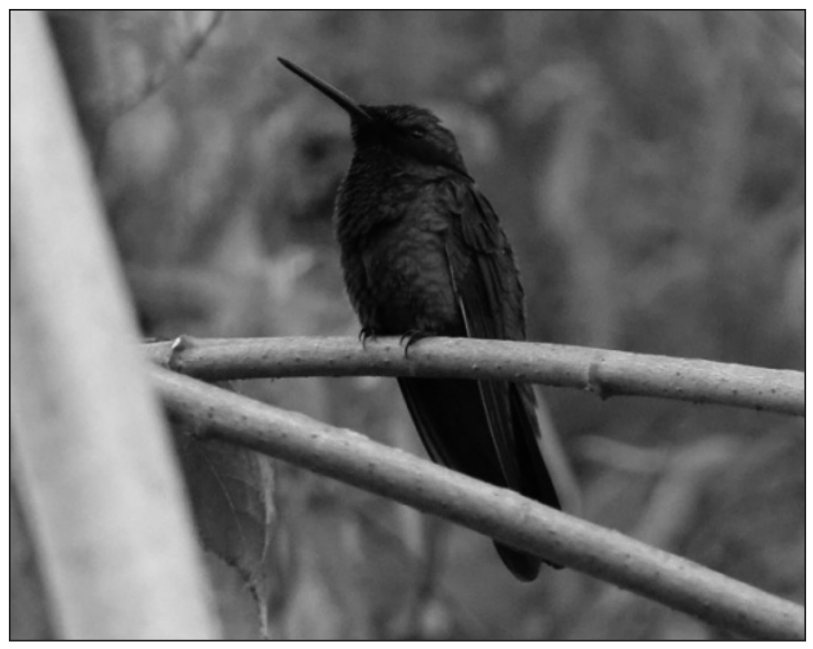

\section{Colibrí coruscans (IJ)}

Quinde herrero TROCHILIDAE

Común

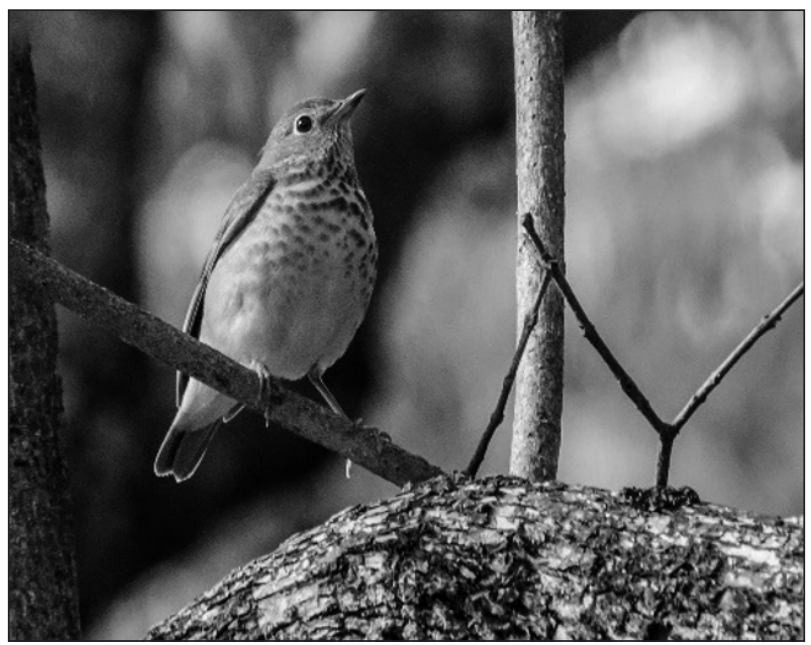

7. Catharus ustulatus (NM)

Zorzal de Swainson TURDIDAE

Raro 


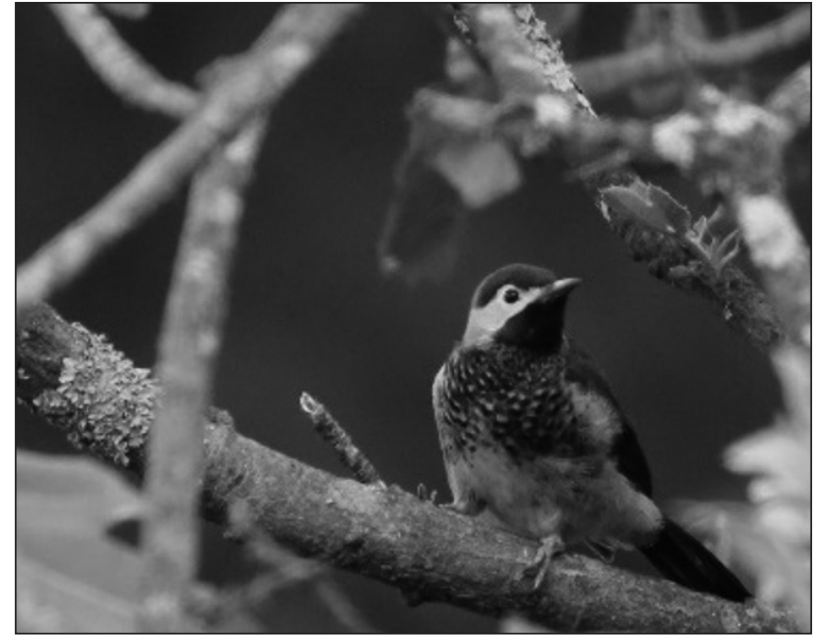

8. Colaptes rivolii (DR)

Carpintero dorsicarmesí PICIDAE

Raro

\section{Referencias}

Abril, A., \& Adela, S. (2011). Diseño de un producto de aviturismo y desarrollo de estrategias para su fortalecimiento en el cantón Patate, provincia de Tungurahua. Escuela Superior Politécnica de Chimborazo.

Albuja, L., Almendáriz, A., Barriga, R., Montalvo, D. Cáceres, F., \& Román, J. (2012). Fauna de Vertebrados del Ecuador. Ecuador: Escuela Politécnica Nacional.

Arteaga, W. (2017). Diversidad de aves del campus universitario de la Universidad Central del Ecuador, Quito, Ecuador. Revista Siembra, $4(1), 172-182$.

Balvanera, P. (2012). Los servicios ecosistémicos que ofrecen los bosques tropicales. Ecosistemas, 21(1-2), 136-147.

Ben-Dalia, S., Collins-Kreiner, N., \& Churchman, A. (2013). Evaluation of an Urban Tourism Destination. Tourism Geographies: An International Journal of Tourism Space, Place and Environment, 15(2), 233-249.

Berget, C. (2006). Efecto del tamaño y la cobertura vegetal de parques urbanos en la riqueza y diversidad de la avifauna de Bogotá, Colombia. Gestión y Ambiente, 9(2), 45-60

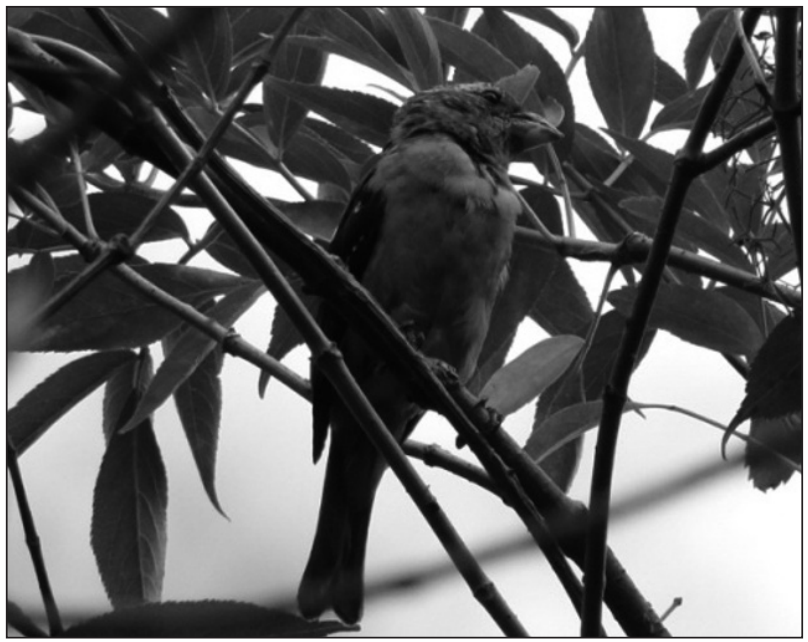

9. Pheuticus chrysogaster (IJ)

Huiracchuro CARDINALIDAE

Común

Blake, J., \& Loiselle, B. (2000). Diversity of birds along an Elevational Gradient in the Cordillera Central, Costa Rica. The Auk: Ornithological Advances, 117(3), 663-686.

Blake, J. G., \& Loiselle, B. A. (2001). Bird assemblages in second-growth and old-growht forest, Costa Rica: Perspectives from mist nets and point counts. The Auk: Ornithological Advances, 118(2), 304-326. https://doi. org/10.2307/4089793

Caula, S., Giner, S., \& De Nóbrega, J. (2010). Aves urbanas: un estudio comparativo en dos parques tropicales con diferente grado de intervención humana (Valencia, Venezuela). FARAUTE de Ciencias y Tecnología, 5(2), 1-13.

Cerezo, A., Robbins, Ch. \& Dowell, B. (2008). Uso de hábitats modificados por aves dependientes de bosque tropical en la región caribeña de Guatemala. Revista Biología Tropical, 57(1-2), 401-419.

Cisneros-Heredia, D., Amigo, X., Arias, D., Arteaga, J., Bedoya, J., Espinosa, S., Montenegro, E., Nazati, G. \& Carrión, J. (2015). Reporte del 1er conteo navideño de aves de Quito, Ecuador. Revista Avances en Ciencias e Ingenierías, 7 (2), B37-B51. 
Colwell, R. K. (2009). Estimates: statistical estimation of species richness and shared species from samples. Version 8.2. Users Guide and Application Published at Httppurloclcorgestimates. Obtenido de: https://doi.org/10.3390/rs70912297

Díaz, I. \& Armesto, J. (2003). La conservación de las aves silvestres en ambientes urbanos de Santiago. Revista Ambiente y Desarrollo de CIPMA XIX, (2), 31-38.

eBird. (2012). eBird: An online database of bird distribution and abundance (web application). Obtenido de:https://doi.org/10.1046/j.10958312.2002.00030.x

Freile, J. F., Brinkhuizen, D. M., Greenfield, P. J., Lysinger, M., Navarrete, L., Nilsson, J., \& Boyla, K. A. (2018). Lista de las aves del Ecuador. Checklist of the Birds of Ecuador. Comité Ecuatoriano de Registros Ornitológicos. Obtenido de: https://ceroecuador.wordpress.com/

García, M., Parra, D., \& Mena, P. (2014). El país de la biodiversidad: Ecuador. Quito: Fundación Botánica de los Andes, Ministerio del Ambiente y Fundación Ecofondo.

Garitano-Zavala, A., \& Gismondi, P. (2003). Variación de la riqueza y diversidad de la ornitofauna en áreas verdes urbanas de las ciudades de La Paz y El Alto (Bolivia). Ecología en Bolivia, 38(1), 65-78. https://doi.org/10.1046/j.13652311.1998.00122.x

Herrera, S., \& Lasso, S. (2014). Belleza y colorido de las aves, una experiencia incomparable en Mindo. Kalpana, 12, 6-12.

Jácome, I., \& Guarderas, L. (2010). Lista preliminar de las aves del cantón Rumiñahui, Pichincha, Ecuador. Ecuador: Corporación para la conservación de la biodiversidad SISAMUNA. Sangolquí, 7.

Jiménez, Moreno, F. \& Mendoza Cuamatzi, R. (2010). Aves urbanas en ciudad universitaria de la BUAP. Revista Elementos, 79, 23-27.

Lara, A. (2015). Diseño de un sistema de conservación para el cantón Rumiñahui. Universidad Técnica Particular de Loja, Loja.

Malagamba-Rubio, A., MacGregor-Fors, I., \& Pineda-López, R. (2013). Comunidades de aves en áreas verdes de la ciudad de Santiago de Querétaro, México. Ornitología Neotropical, 24, 371-386.

Maragliano, R., Marti, L., Ibáñez, L. \& Montalti, D. (2009). Comunidad de aves urbanas de Lava1lol, Buenos Aires, Argentina. Acta Zoológica Lilloana, 53(1-2), 108-114.

Martella, M., Trumper, E., Bellis, L., Renison, D., Giordano, P., Bazzano, G., \& Gleiser, R. (2012). Manual de ecología - Evaluación de la biodiversidad. Reduca (Biología). Serie Ecología, 5(1), 71-115.

McMullan, M., \& Navarrete, L. (2013). Fieldbook of the Birds of Ecuador including the Galápagos Islands. Ecuador: Fundación de Conservación Jocotoco.

Mindo Cloudforest Fundation. (2010). Actualización de la Estrategia Nacional de Aviturismo. Obtenido de: http://suia.ambiente.gob.ec/ documents/783967/890253/Actualización+$\mathrm{de}+\mathrm{la}+$ Estrategia + Nacional + de + Aviturismo. PDF/0f436463-c0c7-43c7-b024-395a7515f1f9

Montenegro-Pazmiño, E. C. (2015). Diversidad de aves en áreas verdes de la ciudad de Quito, Ecuador. Universidad San Francisco de Quito.

Municipio de Rumiñahui. (2016). Línea de tiempo, Rumiñahui 1938-2016. Obtenido de: http:// www.ruminahui.gob.ec/images/d/2016/06/Linea_de_Tiempo.pdf

Ralph, J. C., Geupel, G. R., Pyle, P., Martin, T. E., De Sante, D. F., \& Milá, B. (1996). Manual de métodos de campo para el monitoreo de aves terrestres. General Technical Report PSW-GTR159-Web. Obtenido de: https://doi.org/10.3145/ epi.2006.jan.15

Ramírez-Albores, J. E. (2006). Variación en la composición de comunidades de aves en la Reserva de la Biosfera Montes Azules y áreas adyacentes, Chiapas, México. Biota Neotropica, 6 (2), 1-19. https://doi.org/10.1590/S167606032006000200019

René, E., Marti, L. J., Ibañez, L. M., \& Montalti, D. (2009). Comunidades de aves urbanas de Lava1lol, Buenos Aires, Argentina. Acta Zoológica Lilloana, 53(1-2), 108-114. 
Reyes, I., \& Gutiérrez, J. (2010). Los servicios ambientales de la arborización urbana: retos y aportes para la sustentabilidad de la ciudad de Toluca. Quivera. Revista de Estudios Territoriales, 12 (1), 96-102.

Ridgely, R. S., \& Greenfield, P. J. (2006). Aves del Ecuador: guía de campo (Vol. 1). Quito, Ecuador: Academia de Ciencias Naturales de Filadelfia y Fundación de Conservación Jocotoco.

Sierra, R. (1999). Propuesta preliminar de un sistema de clasificación de vegetación para el Ecuador continental. Quito: Proyecto INEFAN/GEF-BIRF y EcoCiencia. https://doi. org $/ 10.13140 / 2.1 .4520 .9287$

Trávez, J. J., \& Yánez, P. M. (2017). Diversidad y abundancia de avifauna en el campus de la UIDE y el Parque Metropolitano Guangüilta- gua, Distrito Metropolitano de Quito, recomendaciones para su conservación. Boletín Técnico 13, Serie Zoológica, 12-13, 53-69.

Villarreal, H., Álvarez, M., Córdova, S., Escobar, F., Fagua, G., Gast, F., Mendoza, H., Ospina, M., \& Umaña, A. (2006). Manual de métodos para el desarrollo de inventarios de biodiversidad. Colombia: Programa de inventarios de biodiversidad. Instituto de Investigación de Recursos Biológicos Alexander von Humboldt.

Whittaker, R. H. (1972). Evolution and Measurement of Species Diversity. Taxon, 21(2/3), 213251. https://doi.org/10.2307/1218190.

World Trade Organization. (2010). World Trade Report 2010 Trade in natural resources. Obtenido de: https://www.wto.org/english/res_e/publications_e/wtr10_e.htm 